\title{
Remotely Supervised Home-Based Resistance Training and Orthostatic Tolerance in Young Physically Inactive Women During COVID-19
}

Original Research

\begin{abstract}
Caroline Merguerian ${ }^{1}$, Annika C. Smith ${ }^{1}$, Stephen J. Ives ${ }^{1}$
${ }^{1}$ Health and Human Physiological Sciences, Skidmore College, Saratoga Springs, NY/USA

Open Access

Published: November 16, 2021

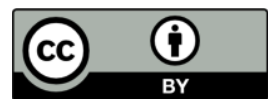

Copyright, 2021 by the authors. Published by Pinnacle Science and the work is licensed under the Creative Commons Attribution 4.0 International License. To view a copy of this license, visit http://creativecommons .org/licenses/by/4.0/

Journal of Exercise and

Abstract

Introduction: Orthostatic intolerance (OI) is the inability of the cardiovascular system to adequately adjust to gravity upon standing, resulting in lightheadedness or syncope. OI can be characteristic of pathology (e.g. postural orthostatic tachycardia syndrome), which disproportionately affect women, and is compounded by inactivity. Thus, we tested the effects of remotely supervised home-based, 4-week resistance training (RT) program vs. control on the orthostatic stress response of previously inactive young females using the NASA Lean Test (NLT).

Methods: Systolic (SBP) and diastolic (DBP) blood pressures, and heart rate (HR) were assessed during the NLT, pre- and post-training, along with fitness and body composition in females randomized to RT or control ( $\mathrm{n}=7$ ).

Results: RT improved fitness (group*intervention, $\mathrm{p}=0.047)$ and fat-mass $(-3.4 \pm 2.9$ vs. $+0.3 \pm 0.7 \Delta \mathrm{lbs}, \mathrm{p}=0.037)$ over control. The NLT significantly altered SBP, DBP, and HR (all, $\mathrm{p}<0.01$ ). For SBP, the interaction of group*intervention approached significance $(\mathrm{p}=0.071)$, lowering SBP in the RT group with the intervention. In DBP, the group*intervention was not significant $(p=0.558)$. For $H R$, the group*intervention interaction approached significance $(\mathrm{p}=0.066)$.

Conclusions: In the current study, short-term remotely-supervised RT using bands tended to decrease SBP and HR during orthostatic stress in young, otherwise healthy, previously inactive females, suggesting improved health but perhaps not orthostatic tolerance.
\end{abstract} Nutrition: 2021, Volume 4 (Issue 4): 20

ISSN: $2640-2572$

Key Words: resistance bands, autonomic nervous system, cardiovascular

Corresponding author: Stephen J. Ives, sives@skidmore.edu

\section{Introduction}

Orthostatic intolerance $(\mathrm{OI})$ can be defined as the inability of the cardiovascular system to appropriately respond to the pull of gravity upon standing from a sitting or supine position due to decreased muscle tone or dysregulation of baroreceptors and chemoreceptors ${ }^{13}$. OI is a hallmark of conditions such as initial and true orthostatic hypotension, vasovagal syncope, and postural orthostatic tachycardia syndrome (POTS) ${ }^{10}$.

Both pharmacological and nonpharmacological methods have been used to combat OI. In previous studies and treatments, pharmacological interventions have been shown to have side effects that may affect the patient's quality of life $^{6}$. Therefore, non-pharmacological interventions are of interest. Studies of non-pharmacological interventions have

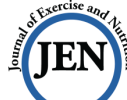


included resistance training, strength training, and the use of compression garments ${ }^{4}$. In order to detect improvements in OI, a classic sit to stand test can be used, or more advanced procedures such as the tilt table test, lower body negative pressure (LBNP), neck pressure/suction collar, or a synchronous combination of two methods can be done. A review of different exercise methods to minimize OI suggested that resistance training can help to manage and decrease OI by increasing core and peripheral muscle tone to prevent the pooling of blood in distal limbs, in both male and female patients ${ }^{2}$.

Patients who experience long-term bed rest and those with postural orthostatic tachycardia syndrome (POTS) or vasovagal syncope, are seen to have exacerbated OI ${ }^{10}$. POTS is a condition that is defined by symptoms of OI, and in some cases can worsen with deconditioning of the skeletal muscle pump and dysregulation of chemoreceptors 9,12 . The OI seen in POTS is mostly found in pediatrics, but sometimes seen in adults, $75-80 \%$ of whom are female 9 Females, in general, are more likely to be diagnosed with OI than male patients (with the exception of pediatric and long-term sedentary patients) ${ }^{9}$. It is interesting to note that, despite higher occurrence in females, studies have failed to show any effect of menstrual cycle phase on OI in females, or on POTS 1,11. Further, previous research suggests that women who are deconditioned are more likely to experience OI than their male counterparts ${ }^{9}$. One method to combat OI is resistance-based exercise, as utilized in previous research ${ }^{12}$. It has been documented that increasing the skeletal muscle pump in the legs using resistance training can aid in preventing venous pooling in female POTS patients specifically ${ }^{4,15}$.

To determine the effectiveness of short-term exercise, a previous 10-week study was conducted on middle aged women to assess the effectiveness of resistance band exercise versus weight machines on body composition. The data showed that fat-free mass increased significantly, and fat mass decreased significantly in both the resistance band and weight machine groups ${ }^{2}$. Thus, at home resistance band exercise has been shown to increase muscle mass over a relatively short length of 10 weeks. However, further research is needed to determine if an even shorter period of exercise can affect fat free mass, crucial for function of the skeletal muscle pump, and if an at home resistance exercise program can produce an increase in orthostatic stress tolerance in young inactive females. No study, to date, has investigated the potential impact of a short-term home-based resistance training using elastic resistance bands on cardiovascular response to orthostasis in previously inactive, but otherwise healthy, young female participants. This is increasingly important in the current global pandemic, which has largely prevented individuals from going to fitness centers to conduct resistance-training. Patient access, even pre-pandemic, has always been an issue.

Accordingly, we propose to study the impact of a 4-week home-based remotely supervised resistance-band training on orthostatic stress response in college-aged physically inactive females. Specifically, we hypothesized that completion of a 4-week home-based supervised resistance-band training program would improve measures of muscular fitness and orthostatic stress response associated with orthostatic challenge. If found to be true this feasibility study, conducted during the COVID-19 pandemic, could provide further evidence of the efficacy of home-based exercise interventions.

\section{Scientific Methods}

\section{Participants}

Healthy, but physically inactive, college-age female participants (aged 18-25) were recruited to participate in the 4-week longitudinal study (Figure 1). Recruiting efforts included creating a poster that was sent out via email to students, and word of mouth. Potential participants were randomly assigned ID numbers to anonymously complete, online, an AHA/ACSM Preparticipation Health/Fitness Screening Questionnaire, and a current activity level questionnaire which were administered via secured Google Forms. Participants were excluded if they reported cardiopulmonary, vascular, metabolic, or neural conditions, had any withstanding injuries that would prevent the participant from completing the fitness assessment and/or the intervention, had medically diagnosed orthostatic intolerance, or were deemed too active participate. During pre-post testing participants were asked to be hydrated, $>2 \mathrm{hr}$ post-prandial, and to avoid supplements, caffeine, and alcohol for $24 \mathrm{hr}$ prior. Participants were asked to keep their diet and sleep habits consistent throughout the study. Participants provided written informed consent prior to participation. The study was approved by the local Institutional Review Board of Skidmore College (IRB\#2102-948). This research was carried out fully in accordance to the ethical standards of the field ${ }^{8}$, and in accordance with the most recent revisions to the Declaration of Helsinki.

Protocol

Participants were randomly assigned to either the control group or intervention group in a parallel design (Figure 1). All participants $(n=7)$ participated in pre-intervention testing (NLT and Fitness Assessment). As this study was 
completed during COVID-19 and in person human subjects research was institutionally prohibited to reduce the risk of COVID-19 transmission, the present study was carried out in an entirely remote manner. Equipment for measuring body weight/body composition (HBF-514c, Body Composition Monitor and Scale, OMRON, Lake Forest, IL) 16, blood pressure/heart rate (1500 Blood Pressure Monitor, Welch Allyn, Skaneateles, NY), as well as a resistance band set (large and mini-resistance bands) were dropped off at each participant's place of residence prior to their scheduled video conference testing appointment with a researcher. The video conference allowed direct supervision by members of the research team while collecting direct measures of blood pressure and heart rate (HR). The drop off was contactless and any shared supplies were sanitized between uses. The participants were first asked to report their age and height. Following recommendations for bioelectric impedance analysis, participants then took their weight, estimated fat mass, and estimated fat free mass using the body composition monitor and scale and reported these values to the researcher. When finished with the scale, the participants were asked to leave it outside their place of residence for contactless pick up.

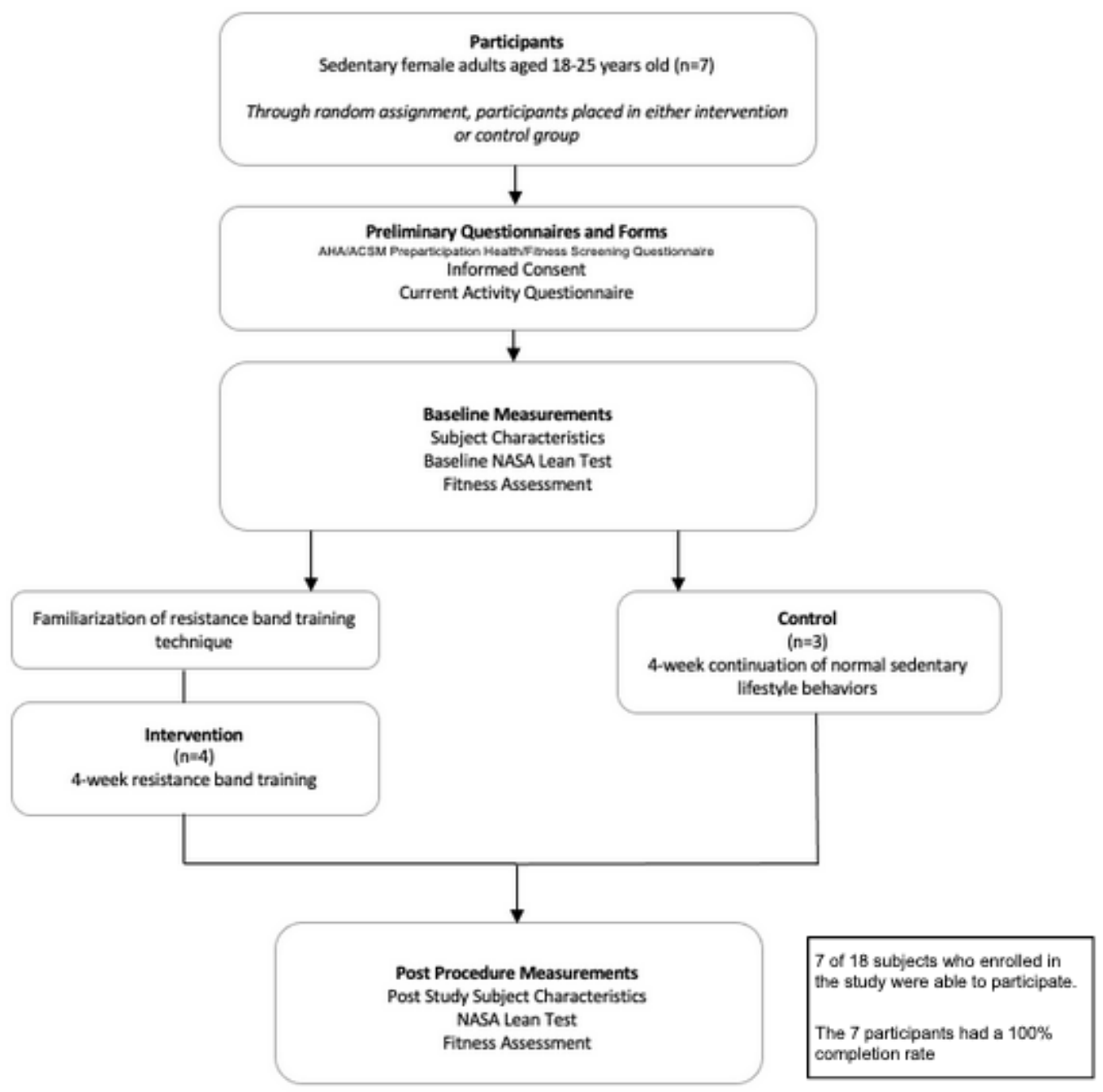

Figure 1. Experimental Overview.

The participants were then instructed on how to use the blood pressure monitor, and then began the NASA Lean Test (NLT), a technique which has previously been used to document impaired hemodynamics in patients with orthostatic intolerance ${ }^{7}$. The NLT starts with participants lying down with no movement for 15 minutes to measure resting blood pressure and heart rate. At the end of the 15 minutes, the participants were instructed to take two blood pressure readings one minute apart, to ensure that it was the true resting measure. To complete the NLT, the participants were asked to stand up and lean against the wall for 10 minutes, with only the shoulder blades touching the wall and feet at least 6 inches from the wall. The participants were asked to be as still as possible, taking a blood pressure reading every 
2 minutes as instructed by the researcher. All data was recorded by the researcher conducting the remote data collection meeting, as the monitor was made visible to the researcher during measurements.

The participants were then guided through the fitness assessment, and instructed on how to perform a banded squat, banded deadlift, banded row, and banded calf raises, using large and mini-resistance bands. They first used the heaviest band recommended by the researcher and moved down in band strength if they could not perform a repetition of the exercise with acceptable form, as assessed by the research team. When the band strength was acceptable, the participant was asked to perform as many unbroken repetitions as possible until they stopped or until stopped by the researcher for technical failure (any break in form). The number of repetitions the participant could complete, and level of resistance band used was recorded by the researcher hosting the session.

After randomization and the pre-intervention testing, control participants $(n=3)$ were instructed to continue their average sedentary daily lifestyle and routine for the duration of the 4-week study. Each participant in the intervention group $(\mathrm{n}=4)$ was scheduled for three live home-based training sessions each week with researchers over video conference, for the duration of the 4-week intervention (12 sessions total). The dynamic warm up, fitness assessment, and resistance training program were developed in consultation with and under the supervision of a National Strength and Conditioning Association, Certified Strength and Conditioning Specialist. Each session had two participants and one researcher who led the exercise routine and monitored the participants. Each session started with a warmup, including stretches, which targeted the muscles being worked (Table 1).

Table 1. Specific warm-up and stretching protocol completed prior to each RT session.

\begin{tabular}{lll}
\hline Day $\mathbf{1}$ (e.g. Monday) & Day 2 (e.g. Wednesday) & Day 3 (e.g. Friday) \\
\hline 2 Sets & 2 Sets & 2 Sets \\
10 glute bridges & 10 air squats & 8 modified push-ups* \\
10 bird dogs (5 each side) & 12 (total) front lunges & 10 toe raises \\
\hline :30 seconds each & $: 30$ seconds each & $: 30$ seconds each \\
Cobra stretch & Single leg sit and reach (each side) & Pike position single leg calf stretch \\
Pigeon stretch (each side) & Butterfly stretch (each side) & (each side) \\
Spiderman stretch (each side) & Spiderman stretch (each side) & Single leg sit and reach (each side) \\
& & Spiderman stretch (each side) \\
\hline
\end{tabular}

*Modified push-ups were done with hands on an elevated surface.

The first session of the week targeted glutes and abs, with banded glute bridges and donkey kicks, and Paloff presses and knee drives (Table 2). The second session focused on quadriceps and hamstrings, with squats and seated leg extensions, and Romanian deadlifts and standing leg curls. The third session of the week targeted the calves and back, with point and flex of the toes and calf raises and seated supinated pull-a-parts and bent over rows. Illustrations of the exercises are presented in Figure 2.

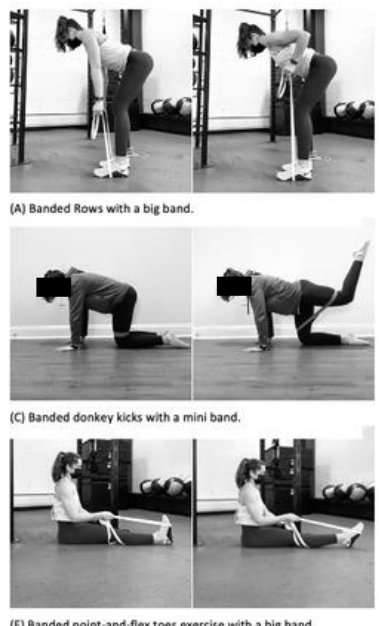

Figure 2. Banded exercises (A-L) performed as a participant would when undergoing RT intervention.

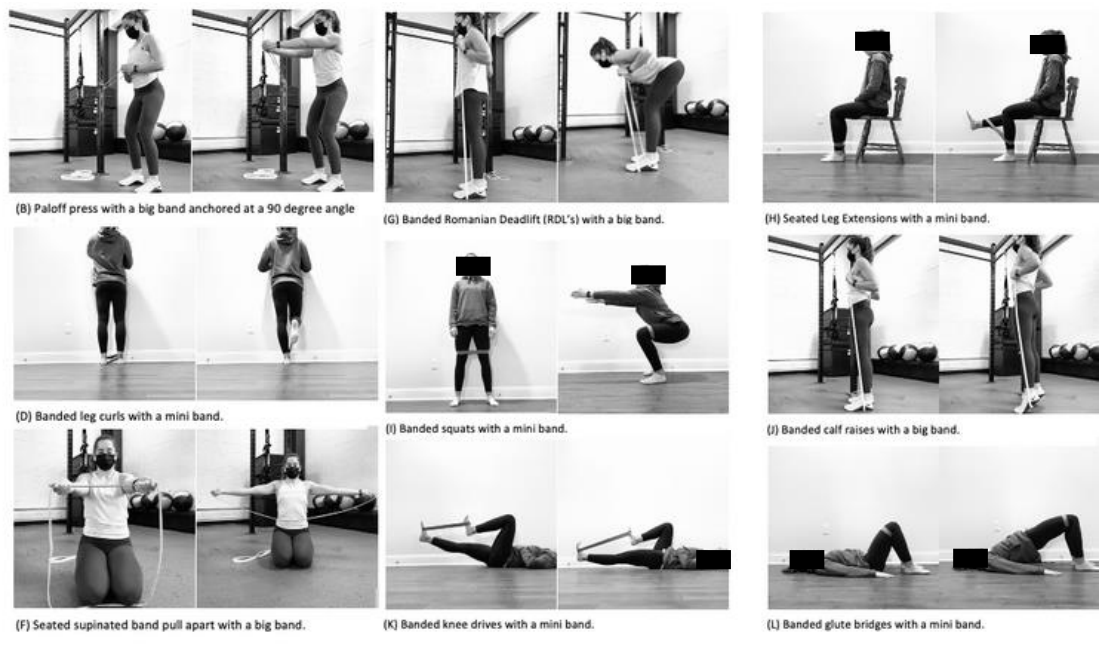


Table 2. Exercise protocol for the intervention group

\begin{tabular}{|c|c|c|c|c|c|c|c|c|c|c|c|c|}
\hline & \multicolumn{3}{|c|}{ Week 1} & \multicolumn{3}{|c|}{ Week 2} & \multicolumn{4}{|c|}{ Week 3* } & \multicolumn{2}{|c|}{ Week 4} \\
\hline Donkey Kicks & 2 & xmini & 10 & 3 & xmini & 10 & 3 & $\mathrm{xmini}$ & 10 & 4 & xmini & 10 \\
\hline Knee Drive & 2 & xmini & 10 & 3 & xmini & 10 & 3 & xmini & 10 & 4 & xmini & 10 \\
\hline Day 2 & $O F F$ & & & & & & & & & & & \\
\hline Day 3 & Sets & Band & Reps & Sets & Band & Reps & Sets & Band & Reps & Sets & Band & Reps \\
\hline RDL's & 2 & xbig & 10 & 3 & xbig & 10 & 3 & xbig & 10 & 4 & xbig & 10 \\
\hline Standing Leg Curl & 2 & xmini & 10 & 3 & xmini & 10 & 3 & xmini & 10 & 4 & xmini & 10 \\
\hline Day 4 & $\mathrm{OFF}$ & & & & & & & & & & & \\
\hline Day 5 & Sets & Band & Reps & Sets & Band & Reps & Sets & Band & Reps & Sets & Band & Reps \\
\hline Point and Flex Toes & 2 & xbig & 10 & 3 & xbig & 10 & 3 & xbig & 10 & 4 & xbig & 10 \\
\hline Calf Raises & 2 & xbig & 10 & 3 & xbig & 10 & 3 & xbig & 10 & 4 & xbig & 10 \\
\hline
\end{tabular}

*Increase resistance $\quad$ xmini $=$ unassigned mini resistance band $\quad$ xbig $=$ unassigned large resistance band

The participants began the intervention with two sets of 10 reps of each exercise, the second week progressed to three sets of 10 reps, the third week increased in band resistance for three sets of 10 reps, and the fourth week increased to 4 sets of 10 reps of each exercise at the increased resistance from week three. Participants had at least one day of rest between each session. Minimum compliance was set at participants completing 75\% of sessions.

Post-Intervention Testing (NLT and Fitness Assessment): At the end of the 4-week intervention, the participants in both the control and the intervention groups participated in post-intervention testing at the same time of day as baseline. The same protocol used in the pre-intervention testing was used to drop off the scale at each participant's place of residence. Each participant was scheduled for a video conference testing session with a researcher. Weight, fat mass, and fat free mass were taken using the OMRON Body Composition Monitor and Scale HBF-514c and recorded by the researcher hosting the testing session. The participants then participated in the NLT. The researcher recorded resting blood pressure and heart rate, and then blood pressure and heart rate every 2 minutes during the 10 minute wall stand. The participants then participated in the same fitness assessment as they had done pre-intervention.

\section{Statistical Analysis}

Data were analyzed using open-source software (JASP v. 0.13.1.0, Netherlands). Independent samples t-tests were used to compare groups at baseline. Two-way $(2 \mathrm{x} 2)$ mixed model analysis of variance (ANOVA) was used to examine changes over the intervention period (pre-post), between groups, and the potential interaction of group over the 4 weeks. Change over the intervention was also examined using independent samples t-tests. A multivariate $(2 \times 2 \times 6)$ mixed model ANOVA was used to detect differences between the RT and control groups (group), pre- and postintervention (intervention), and with time during the NLT (time). Tests for the assumptions of normality were conducted and if a significant violation was found, an appropriate adjustment to the degrees of freedom was made. Significant differences were followed up using appropriate post hoc pairwise comparisons, as necessary. Alpha was set at $\mathrm{p}<0.05$. All data are presented as means \pm standard deviation.

\section{Results}

Subject characteristics measured included age (years), height $(\mathrm{cm})$, body weight $(\mathrm{kg})$, fat mass $(\%)$, and free fat mass $(\%)$, where body weight, fat mass, and free fat mass were measured both pre- and post-intervention. Participants were, on average, $161.8( \pm 3.2) \mathrm{cm}$ tall, $67.5( \pm 18.6) \mathrm{kg}$ in mass, and $21.0( \pm 0.8)$ years old. There were no significant differences in age, height, weight, fat mass (FM \%), or fat-free mass (FFM \%) between or within subjects (Table 3). Resting baseline measurements of SBP $(\mathrm{mmHg}), \mathrm{DBP}(\mathrm{mmHg})$, and $\mathrm{HR}(\mathrm{bpm})$ in all participants were analyzed to determine any significant differences. There were no significant differences among participants for pre and post intervention SBP and HR. However, there was a significant difference in pre-intervention DBP measures when comparing control and resistance training $(\mathrm{RT})$ groups $(\mathrm{p}=0.035$, Table 3$)$. There were no significant findings when analyzing post-intervention DBP. The average change in fat mass was greater with RT $(-3.4 \pm 2.9$ vs. $+0.3 \pm 0.7 \Delta \mathrm{lbs}, \mathrm{p}=0.037)$, while change body 
weight tended to be greater with RT $(-4.8 \pm 6.7$ vs. $+2.1 \pm 3.6 \Delta \mathrm{lbs}, \mathrm{p}=0.071)$, but change in fat-free mass was not different $(-0.75 \pm 4.5$ vs. $+1.1 \pm 2.1 \Delta \mathrm{lbs}, \mathrm{p}=0.259)$.

Table 3. Mean \pm standard deviation of baseline measurements pre- and post-intervention in both the control and resistance training (RT) groups.

\begin{tabular}{lcccc}
\hline & Control-Pre & RT-Pre & Control-Post & RT-Post \\
\hline Age (years) & $21.33 \pm 0.58$ & $20.75 \pm 0.96$ & - & - \\
Height (cm) & $162 \pm 3.8$ & $162 \pm 2.9$ & - & - \\
Weight (kg) & $60.56 \pm 12.98$ & $72.74 \pm 22.17$ & $61.53 \pm 14.61$ & $72.52 \pm 22.41$ \\
FM (\%) & $32.93 \pm 7.33$ & $40.13 \pm 9.26$ & $32.63 \pm 6.90$ & $39.18 \pm 10.88$ \\
FFM (\%) & $28.13 \pm 2.10$ & $25.00 \pm 2.38$ & $28.40 \pm 1.61$ & $25.43 \pm 4.27$ \\
Resting SBP (mmHg) & $98 \pm 10$ & $118 \pm 14$ & $103 \pm 10$ & $111 \pm 10$ \\
Resting DBP (mmHg) & $59 \pm 4$ & $71 \pm 7 *$ & $63 \pm 8$ & $72 \pm 6$ \\
Resting HR (bpm) & $71 \pm 10$ & $78 \pm 7$ & $68 \pm 6$ & $70 \pm 6$ \\
\hline
\end{tabular}

FM, fat mass; FFM, fat-free mass; SBP/DBP, systolic/diastolic blood pressure. ${ }^{*} \mathrm{p}<0.05$ Control vs. RT at baseline.

The control and RT groups underwent a fitness assessment to analyze any change in muscular strength pre- to postintervention. A 2-way ANOVA showed that that there was an effect of intervention $(p=0.029)$, effect of intervention*group $(p=0.047)$, and an effect of exercise for band strength $(p<0.001$; Table 4$)$. There was an effect of exercise $(\mathrm{p}=0.005)$, along with exercise* group for repetitions completed ( $\mathrm{p}=0.002$; Table 4$)$. If looking at a composite score of reps $\mathrm{x}$ band strength, and the change over the intervention, the RT group had a greater change in composite score in squat (61 vs. 20$)$, RDL (7 vs. 0), Rows (27 vs. 12), and calf raises (14 vs. -2$)$. The RT group had 100\% compliance with attending the training sessions and 100\% adherence in completing the program.

Table 4. Mean \pm standard deviation of band resistance and repetitions (reps) for the fitness assessment in control $(\mathrm{n}=3)$ and resistance training $(\mathrm{RT})(\mathrm{n}=4)$ groups pre- and post-intervention.

\begin{tabular}{llcccc}
\hline Exercise & Group & Pre-Band & Reps & Post-Band & Reps \\
\hline Squat & Control & $5 \pm 1$ & $7 \pm 6$ & $5 \pm 0$ & $11 \pm 4$ \\
& RT & $4 \pm 1$ & $11 \pm 6$ & $5 \pm 0$ & $21 \pm 12$ \\
\hline RDL & Control & $2 \pm 1$ & $8 \pm 2$ & $2 \pm 0$ & $8 \pm 4$ \\
& RT & $2 \pm 1$ & $10 \pm 3$ & $3 \pm 1$ & $9 \pm 8$ \\
\hline Rows & Control & $2 \pm 1$ & $8 \pm 4$ & $2 \pm 1$ & $14 \pm 3$ \\
& RT & $3 \pm 1$ & $5 \pm 3$ & $3 \pm 1$ & $14 \pm 4$ \\
\hline Calf Raises & Control & $2 \pm 1$ & $19 \pm 7$ & $2 \pm 0$ & $18 \pm 6$ \\
& RT & $2 \pm 1$ & $11 \pm 2$ & $3 \pm 0$ & $12 \pm 9$ \\
\hline
\end{tabular}

Band Strength Scoring: X-Light=1, Light $=2$, Medium=3, Heavy=4, X-Heavy=5. Romanian deadlift (RDL)

SBP, DBP, and HR in both control and RT groups were all analyzed within the NLT using a MANOVA. When analyzing SBP, it was found that there was a significant effect of time $(\mathrm{p}<0.001)$ and time ${ }^{*}$ group $(\mathrm{p}=0.001$, Figure 3$)$. While not significant, the effect of intervention* ${ }^{*}$ group for SBP approached significance $(\mathrm{p}=0.071$, Figure 3$)$. There was a significant effect of time $(\mathrm{p}<0.001)$ and time*group for DBP measurements $(\mathrm{p}=0.004$, Figure 3$)$, however no significant effect of intervention (pre-post) or intervention*group was observed. For HR measurements there was only a significant effect of time $(\mathrm{p}<0.01$, Figure 4). However, there were close to significant findings for the effect of intervention*group in HR ( $\mathrm{p}=0.066$, Figure 4). There were no significant findings between subjects for any of the variables measured. 
Group: Control

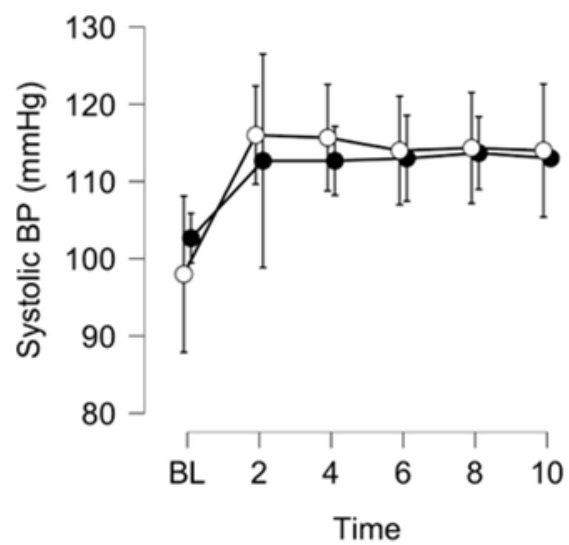

Group: Control

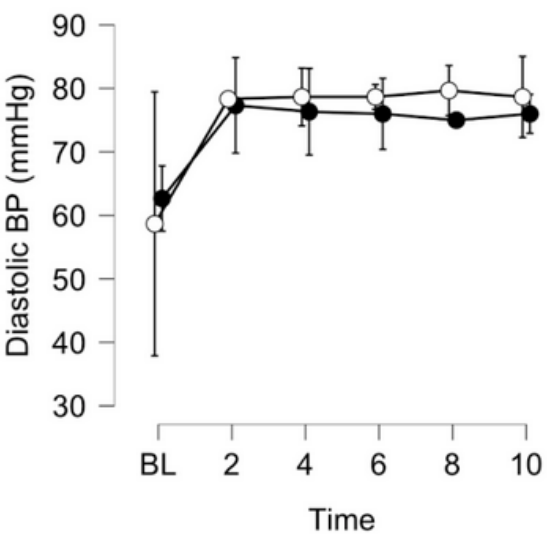

Group: RT
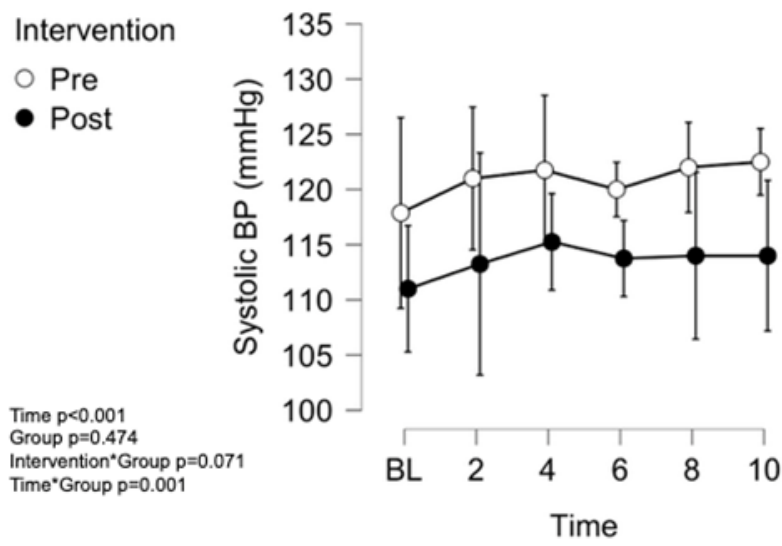

Intervention

- Pre

- Post

Group: RT

Intervention

Pre

- Post

Figure 3. Control ( $\mathrm{n}=3$ ) and Resistance Training $(\mathrm{RT}, \mathrm{n}=4)$ Groups Systolic (top) and Diastolic (bottom) Blood Pressures at Baseline (BL) and During the NASA Lean Test Pre- and Post-Intervention. Data are means \pm SD.
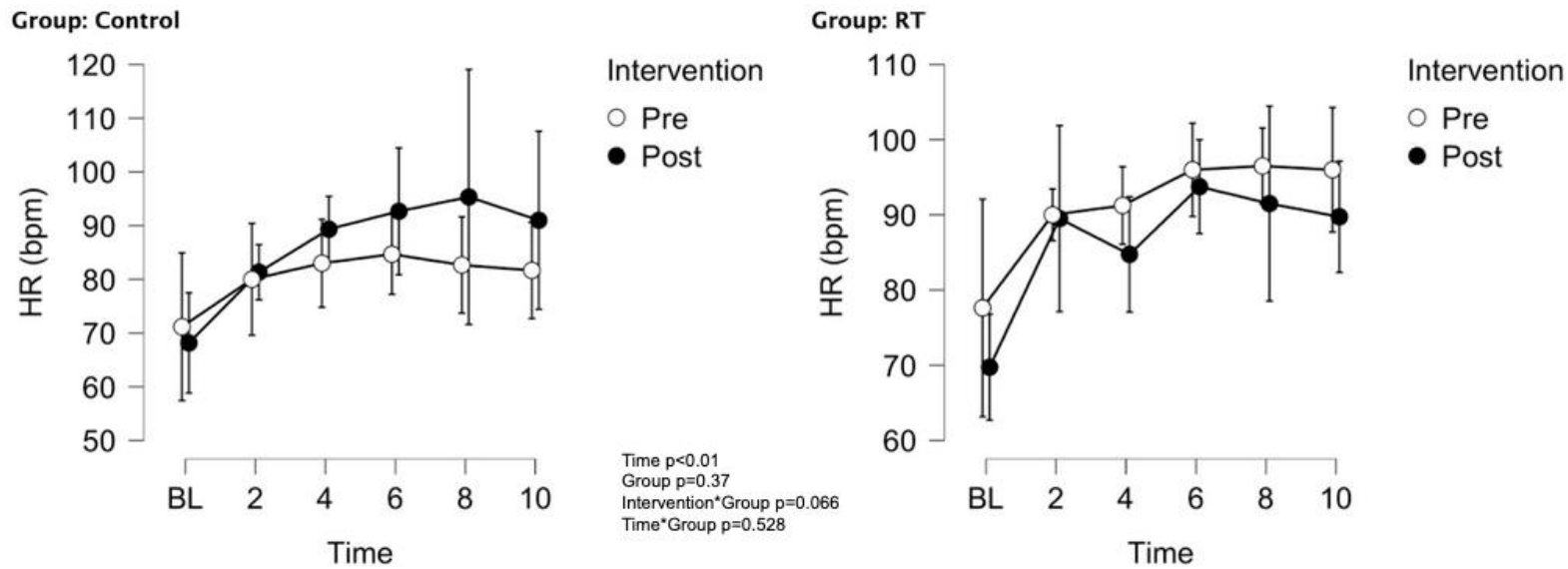

Figure 4. Control $(\mathrm{n}=3)$ and Resistance Training $(\mathrm{RT}, \mathrm{n}=4)$ Groups Heart Rate (HR, beats/min, bpm) at Baseline (BL) and During the NASA Lean Test Pre- and Post-Intervention. Data are mean \pm SD.

\section{Discussion}

The goal of this study was to investigate if the non-pharmacological method of resistance band training could increase tolerance to orthostatic stress during a NASA Lean Test in previously inactive college age females, even if conducted remotely during the COVID-19 pandemic. It was hypothesized that the resistance band training protocol would 
increase the participants ability to withstand orthostatic stress during the test. We found that resistance training group heart rate and systolic blood pressure (SBP) response to the orthostatic challenge was disparate over the intervention, though in those who completed RT protocol actually had, on average, lower blood pressure, perhaps indicating improved cardiovascular health, but not orthostatic tolerance, per se, but more work on this topic is warranted. Finally, this study provides evidence of the efficacy of remotely supervised home-based resistance training intervention, a key finding in the ongoing COVID-19 pandemic.

A possible explanation for the tendency for a decrease in heart rate (HR) and SBP in the intervention group could be due to increased efficiency of the heart, as a result of regular exercise. Previous research has found that exercise training increases plasma volume, ventricular filling and/or contractility of the left ventricle, increasing stroke volume, which in turn would allow for a reduction in $\mathrm{HR}^{3}$. While the intervention was only four weeks, it is possible that the regular exercise caused a small but perhaps effective increase in the intervention participants stroke volume via plasma volume and/or cardiac hemodynamics (e.g. contractility \&/or relaxation). Theoretically, an increase in stroke volume (SV) would support the decrease in heart rate (HR), allowing for a stable cardiac output (CO) given the relationship of CO $=\mathrm{HR} \times \mathrm{SV}$. In this case, the increase in stroke volume would support cardiac output (CO), while allowing the heart rate to decrease, as the heart would not need to pump as rapidly to support the same cardiac output.

The decrease in SBP may reflect improved cardiovascular health, but also may be explained by the relationship between mean arterial pressure, $\mathrm{CO}$ and total peripheral resistance (MAP $=\mathrm{CO} \times \mathrm{TPR}$ ). With the decrease in heart rate, a small decrease in $\mathrm{CO}$ (even if it was supported by increased SV) may have also occurred, creating a lower blood pressure as well. Another possible explanation would be that upon standing, all individuals experience a decrease in central venous pressure, stroke volume, cardiac output, and mean arterial pressure ${ }^{14}$. Baroreflex-mediated increases in heart rate and total peripheral resistance have to occur to counteract these decrements in blood pressure ${ }^{14}$. In our study, the decrease in heart rate in the intervention group post-intervention may mean that upon standing, stroke volume may have decreased less in the RT group than experienced pre-training, meaning heart rate did not have to increase as greatly to regulate these drops. Alternatively, resistance training-mediated improvement in baroreflex sensitivity ${ }^{5}$, via total peripheral resistance, perhaps due to the skeletal muscle pump might have supported mean arterial pressure versus central hemodynamic responses. Therefore, one interpretation of the impact of the RT intervention might suggest improved orthostatic tolerance. However, by design due to the remote nature of the data collection, the orthostatic challenge was not severe, such that both pre and post intervention, no participants had to end the test early, or felt any pre-syncopal symptoms. Thus, a more intense orthostatic challenge with measures of central hemodynamics might provide greater insights into the reductions in HR with orthostatic challenge after completing a resistance training program.

Interestingly, while a decrease in blood pressure, during the NLT is not the goal for individuals suffering from orthostatic hypotension, this study does show promise that resistance band training did not appear to overtly reduce orthostatic tolerance in inactive young women, but may afford a more efficient means of regulating blood pressure and avoiding pre-syncopal symptoms or syncope. This is key because women who are deconditioned are more likely to experience orthostatic intolerance than are males ${ }^{9}$. Further, it has been found that patients with POTS usually have an, on average, smaller cardiac dimensions coupled with reduced blood volume ${ }^{3}$. In a study that investigated cardiac adaptations to training, exercise training was found to increase contraction force of the heart, ventricular filling, as well as blood volume in patients with POTS ${ }^{3}$. Therefore, an increase in stroke volume through increased contractility and ventricular filling would be beneficial for individuals with disorders such as POTS. Resistance training-induced improvements in the skeletal muscle pump in the legs could also aid in preventing venous pooling in female POTS patients specifically ${ }^{4,15}$. More broadly, resistance-based exercise might improve orthostatic tolerance, as documented in previous research ${ }^{12}$, through enhanced total peripheral resistance response to orthostatic challenge, perhaps mediated via greater skeletal muscle pump and/or sympathetic vasoconstriction could also better support blood pressure and orthostatic tolerance.

Due to the COVID-19 pandemic, and obvious risk of viral transmission the safest way to conduct human research was to do so nearly completely virtually, using video conference technology (specifically Google Meet), and modest procedures and instrumentation that were capable of being administered by participants themselves in safe manner. Though the study was designed to be conducted virtually, as we could do no in laboratory testing, it presents both as a limitation and but also as a potentially underutilized opportunity. Future researchers should more strongly consider remote training as adherence in this study was high and our outcome measures of strength were significantly improved. 
Though imperfect, the present study highlights the potential for undergraduate research projects to be carried out in whole or partially remote manner, perhaps increasing research opportunities for students.

The following are presented as experimental considerations. The sample size was also modest $(n=7)$, due to institutional restrictions on recruiting only Skidmore students, who had to be female, physically inactive, and meet the criteria of the study (e.g. healthy and inactive) during a pandemic. Many students were often under quarantine restrictions and/or were experiencing chronic stress with the pandemic, further reducing participation rates. A larger sample size would also be beneficial to determine any correlations between enhanced muscle strength or fat-free mass with resistance band training and increased orthostatic stress tolerance. The pandemic and forced remote nature of the current work, prevented use of more strenuous approach to test orthostatic tolerance, such as the lower body negative pressure (LBNP) chamber or tilt table. No participants experienced any orthostatic intolerance (e.g. syncope), thus we cannot know if the intervention truly impacted orthostatic tolerance, only the cardiovascular response to the orthostatic stress imposed. Relatedly, we were unable to document the possible training-induced changes in central hemodynamics (SV, HR, CO, MAP) typically measured with a Finometer, or cerebral and peripheral microvascular responses often measured via near infrared spectroscopy during the orthostatic stress. The study was intentionally limited by time, as to maintain compliance, and avoid potential future institutional shut downs as a result of COVID-19. Therefore, follow up studies utilizing an exercise intervention longer than 4-weeks might alter the findings.

\section{Conclusions}

The main findings of the current study were that home-based supervised resistance training with bands, increased muscular strength/endurance, resulted in more favorable changes in body weight and body composition versus the control group. RT reduced systolic blood pressure, and tended to reduce HR during the NLT as compared to the control group. This decrease in SBP and heart rate could mean an increase in efficiency of the heart, which would be beneficial in previously inactive women, and potentially those suffering from orthostatic intolerance. Further work is warranted on the effects of resistance band training on orthostatic tolerance in young inactive women, as such training could prove beneficial.

\section{Acknowledgements}

We would like to acknowledge and thank the individuals who dedicated their time and effort to participating in the study. Their commitment to attending exercise sessions and maintaining an unwavering work ethic made the study possible. We thank the Human Health and Physiology department for supplying us with the necessary equipment to run our study. We would also like to thank Carolyn E. Blackman for her assistance in the study. The authors have no funding sources to report or conflicts of interest to disclose.

\section{References}

1. Claydon V, Younis N, Hainsworth R. Phase of the menstrual cycle does not affect orthostatic tolerance in healthy women. Clin Auton Res 16(2): 98-104, 2006.

2. Colado JC, Triplett NT. Effects of a short-term resistance program using elastic bands versus weight machines for sedentary middle-aged women. J Strength Cond Res 22(5): 1441-1448, 2008.

3. Fu Q, VanGundy TB, Galbreath MM, Shigeki S, Jain M, Hastings JL, Bhella PS, Levine BD. Cardiac origins of the postural orthostatic tachycardia syndrome. J Am Coll Cardiol 55(25): 2858-2868, 2010.

4. Fu Q, Levine BD. Exercise and non-pharmacological treatment of POTS. Auton Neurosci 215: 20-27, 2018.

5. Gomes MFP, Borges ME, Rossi VA, deMoura EOC, Medeiros A. The effect of physical resistance training on baroreflex sensitivity of hypertensive rats. Arq Bras Cardiol 108(6): 539-545, 2017.

6. Joyner MJ. Standing up for exercise: should deconditioning be medicalized? J Physiol 590(15): 3413-3414, 2012.

7. Lee J, Vernon SD, Jeys P, Ali W, Campos A, Unutmaz D, Yellman B, Bateman L. Hemodynamics during the $10-$ minute NASA Lean Test: evidence of circulatory decompensation in a subset of ME/CFS patients. J Transl Med 18(1): 314, 2020.

8. Navalta JW, Stone WJ, Lyons TS. Ethical issues relating to scientific discovery in exercise science. Int J Exerc Sci 12(1): 1-8, 2019.

9. Stewart JM. Chronic orthostatic intolerance and the postural tachycardia syndrome (POTS). J Pediatr 145(6): 725 730, 2004.

10. Stewart JM, Boris JR, Chelimsky G, Fischer PR, Fortunato JE, Grubb BP, Heyer GL, Jarjour IT, Medow MS, Numan MT, Pianosi PT, Singer W, Tarbell S, Chelimsky TC. Pediatric disorders of orthostatic intolerance. Pediatrics 141(1): e20171673, 2018. 
11. Stickford ASL, VanGundy TB, Levine BD, Fu Q. Menstrual cycle phase does not affect sympathetic neural activity in women with postural orthostatic tachycardia syndrome. J Physiol 593(9): 2131-2143, 2015.

12. Taneja I, Medow MS, Clarke DA, Ocon AJ, Stewart JM. Baroreceptor unloading in postural tachycardia syndrome augments peripheral chemoreceptor sensitivity and decreases central chemoreceptor sensitivity. Am J Physiol Heart Circ Physiol 301(1): 173-179, 2011.

13. van Lieshout JJ. Exercise training and orthostatic intolerance: a paradox? J Physiol 551(2): 401, 2003.

14. van Lieshout JJ, Pott F, Madsen PL, van Goudoever J, Secher NH. Muscle tensing during standing: effects on cerebral tissue oxygenation and cerebral artery blood velocity. Stroke 32(7): 1546, 2001.

15. Wieling W, van Lieshout JJ, Hainsworth R. Extracellular fluid volume expansion in patients with posturally related syncope. Clin Auton Res 12(4): 242-249, 2002.

16. Williams RD, Barnes JT, Pujol TJ. Comparison of body composition assessment techniques in a non-athletic, nonobese young adult population. J Strength Cond Res 24(1): 2010.Johnson J, Johnson B, Stella CA. Maximum distance and performance measures in football. J Exerc and Nutr. 2017;1(1). Doi: 101010202012. 